\title{
Lossless Watermarking Considering the Human Visual System
}

\author{
Mohammad Awrangjeb and Mohan S Kankanhalli \\ Department of Computer Science \\ School of Computing \\ National University of Singapore, Singapore 117543 \\ \{mohamma1, mohan\}@comp.nus.edu.sg
}

\begin{abstract}
Due to quantization error, bit-replacement, or truncation, most data embedding techniques proposed so far lead to distortions in the original image. These distortions create problems in some areas such as medical, astronomical, and military imagery. Lossless watermarking is an exact restoration approach for recovering the original image from the watermarked image. In this paper we present a novel reversible watermarking technique with higher embedding capacity considering the Human Visual System (HVS). During embedding we detect the textured blocks, extract LSBs of the pixel-values from these textured blocks considering the HVS and concatenate the authentication information with the compressed bit-string. We then replace the LSBs of the textured blocks considering the HVS with this bit-string. Since we consider the HVS while extracting LSBs and embedding the payload, the distortions in the resulting watermarked image are completely reversible and imperceptible. We present experimental results to demonstrate the utility of our proposed algorithm.
\end{abstract}

\section{Introduction}

Content authentication of multimedia data like images is becoming more and more important in various fields such as law enforcement, medical imagery, astronomical research, etc. One of the most important requirements in this field is to have the original image during judgment to take the right decision. Cryptographic techniques based on either symmetric key or asymmetric key methods cannot give adequate security and integrity for content authentication. Chiefly, (i) people sharing the secret key can produce false links between the header and its corresponding image in the medical data base, (ii) public key infrastructure is essential, (iii) common image fileformat is required, (iv) compression-decompression results in information loss, are problems with such techniques. Lossless watermarking, a type of fragile watermarking, is the process that allows exact recovery of the original image by extracting the embedding information from the watermarked image. If the watermarked image is deemed to be authentic, that means no single bit of the watermarked image is changed after embedding the payload to the original image. Some authors use distortion free, invertible, reversible, erasable watermarking interchangeably for lossless watermarking. This technique (lossless watermarking) embeds 
secret information with the image so that the embedded message is hidden, invisible and fragile. Any attempt to change the watermarked image will make the authentication fail.

Fridrich et al. [3] and Honsinger et al. [2] use the spread spectrum approach to add the information payload with the host signal. The methods are robust to a wide range of distortions, but due to use of modulo arithmetic salt-and-pepper noise are introduced and the embedding capacity is low. Celik et al. [1] propose a simple reversible data-hiding algorithm having high embedding capacity. It searches the whole image and finds out the lower L-levels of pixel-values (say for $\mathrm{L}=4$, pixel with values $0,1,2,3$ ) to obtain enough space after compression. The main problem with this method is that all images do not offer enough features (pixel-values) at low levels for lossless compression. So, as L becomes larger, distortions become higher and perceptually visible. The lossless compression and encryption of bit-plane method in [3] offers high embedding capacity with strength equivalent to the security offered by cryptographic methods. But noisy images and high payload force the embedding of the message in higher bit-planes and hence distortions become visible. The invertible authentication for JPEG images [3], [4], [10] is a fast algorithm offering high embedding capacity; but artifacts becomes visible for JPEG images with high quality factor. The RS-vector lossless data embedding method in [4], [7], [9] divides the image into disjoint groups (Regular, Singular, and Unusable). The whole image is scanned and Regular-Singular groups are checked whether there is a need to apply the flip operation while embedding information. Though this method involves low and invertible distortions, the capacity is not very high. Tian in [5], [17] proposes a high capacity watermarking algorithm based on difference expansion. This method involves no compression-decompression process of selected image features; but there is significant degradation of image quality due to bit-replacement. Macq et al. [12] proposed an original circular interpretation of bijective transformations as a solution to fulfill all quality and functionality requirements of lossless watermarking. In his first work [18] Macq proposes an additive method that is criticized by him in [19] for having 'salt-and-pepper' visual artifacts due to wrapped around pixels. In [19], [12] Macq et al. propose a modification that solves this problem. It essentially follows the idea of patchwork algorithm [20]. This method helps to convey embedded message from lossless environment to lossy environment and offers high capacity; but the visual quality of watermarked image is not good. Xuan et al. [13] propose a lossless algorithm based on integer wavelet transform. This method offers high embedding capacity but distortions are easily visible if multiple or higher bit-planes are used for embedding. Shi et al. [15] propose a high capacity distortion-free data hiding technique for palette image. They also propose a reversible data hiding technique [14] based on shifting of histogram. In this method though distortions are low the embedding capacity is limited by the frequency of most frequent pixel value in the image.

Celik et al. [1] classify the lossless watermarking techniques in two types. In the first type of algorithms [2], [3], during encoding a spread spectrum signal corresponding to the information payload is superimposed (added) on the host signal. During decoding the payload (watermark signal) is removed (subtracted) from the watermarked image in the restoration step. The advantage of these algorithms is the use of spread spectrum signal as payload increases robustness. But the disadvantages 
are they (i) create salt-and-pepper artifacts in watermarked image due to modulo arithmetic, and (ii) offer very limited capacity.

In the second type of algorithms [1], [4], [5], some features (portions) of the original image are replaced or overwritten with the watermark payload. The original portions of the image that will be replaced by watermark payload are compressed and passed as a part of the embedded payload during embedding. During the decoding process this compressed payload-part is extracted and decompressed. Thus the original image is recovered by replacing the modified portions with these decompressed original features. The advantage of second type of algorithms is they do not suffer from salt-and-pepper artifacts. The disadvantages are: (i) they are not as robust as the first type of algorithms, and (ii) the capacity found, though higher than the first type of algorithms offer, is still not good enough. However, algorithms of second type are better than first type for content authentication where fragility is more important than robustness.

This paper presents a novel reversible watermarking technique that considers the Human Visual System (HVS) [6]. The proposed method offers completely distortioninvisible watermarked image with higher embedding capacity. We find out textured blocks from the original image and embed information in these textured blocks considering the HVS. We keep a pixel of a textured block as is if the corresponding Just Noticeable Distortion (JND) value of the pixel does not allow to change the pixel value by the required amount. Finally when we complete the embedding we do not leave any pixel distorted out of a certain limit determined by its JND value. Hence, the distortions in the watermarked image become completely invisible. However we can get higher capacity to pass the authentication information as well as the side information (helps during decoding) by compressing the bit-string of LSBs using a simple lossless compression algorithm, such as arithmetic coding [8]. Extra capacity can be used to embed necessary information as required. Our implementation and experimental results show that the proposed algorithm fulfills the requirements (invisible distortions and higher capacity) of lossless watermarking technique and is better than other existing algorithms in the literature.

The rest of the paper goes as follows: section 2 represents the general principle of lossless or reversible data embedding technique, and the Human Visual System (HVS), section 3 presents our proposed algorithm, section 4 presents experimental results we have found and decision by comparing the proposed algorithm with other methods published so far, and finally section 5 concludes with advantages and disadvantages of proposed algorithm.

\section{The General Principle of Reversible Data Hiding and HVS}

First we present the general principle [7] of reversible data hiding technique in section 2.1. Then we describe the Human Visual System [6] (in section 2.2) that we use in the proposed algorithm. 


\subsection{The General Principle of Reversible Data Hiding}

The general principle of reversible data hiding is that for a digital object (say a JPEG image file) $I$, a subset $J$ of $I(J \subset I)$ is chosen. $J$ has the structural property that it can be easily randomized without changing the essential property of $I$, and it offers lossless compression itself to have enough space (at least 128 bits) to embed the authentication message (say hash of $I$ ). During embedding $J$ is replaced by the authentication message concatenated with compressed $J$. If $J$ is highly compressible only a subset of $J$ can be used. During the decoding process authentication information together with compressed $J$ is extracted. This extracted $J$ (compressed) is decompressed to replace the modified features in the watermarked image; hence the exact copy of the original image is found.

However when we do embedding for reversible watermarking we have the following points to consider: (i) embedding method should find enough capacity to embed sufficient amount of message, (ii) there should be no expansion of image size due to embedding, (iii) embedded message should be useful, (iv) distortions in original image are kept as low as possible, so artifacts are imperceptible, (v) watermarked image should be fragile to attempt to any kind of change, (vi) encryption with a secret key can increase security and integrity of image data.

\subsection{The Human Visual System}

The most important requirement of lossless watermarking is that any difference (distortion) between the original image and the watermarked image should be perceptually invisible. The Human Visual System (HVS) is the factor that can be exploited to achieve this requirement. But until now there is no lossless watermarking method proposed has utilized the properties of HVS. HVS essentially results in the fact that each pixel value of an image can be changed only by a certain amount without making any perceptible difference to the image quality. This limit is called the just noticeable distortion or JND level. If we keep the distortion to a pixel not out of the limit defined by its JND level the degradation of the pixel is imperceptible in the watermarked image [6].

We divide the original image into $8 \times 8$ blocks, and then take the Discrete Cosine Transfer (DCT) of each $8 \times 8$ block. In a block with abrupt changes between adjacent pixels, the signal energy tends to be concentrated in the $A C$ coefficients. The equation (1) gives the energy, $E_{A C}$, in the $A C$ coefficients, and the equation (2) gives the maximum energy, $E_{\max }$. The maximum energy is found when the adjacent pixels have maximum and minimum permissible gray values. By using $E_{\max }$ as the normalization factor we measure the roughness level $R_{b}$ for block b (equation 3 ).

$$
\begin{gathered}
E_{A C}=\log \sum\left(x_{i, j}^{2}-x_{0,0}^{2}\right), 0 \leq i, j<8 . \\
E_{\max }=\log \left(\frac{G}{2}\right)^{2} .
\end{gathered}
$$




$$
R_{b}=\frac{E_{A C}}{E_{\max }} .
$$

Where $x_{i j}$ is the $(\mathrm{i}, \mathrm{j})$ DCT coefficient of a block, $G$ is the maximum permissible gray value (for 8-bit image it is 255), and we use log for range compression. The range of $R_{b}$ is uniformly divided into 8 subgroups and depending on the value of $R_{b}$ each block is given a block distortion index $I_{b}, I_{b} \in\{1,2, \ldots, 8\}$. The fluctuation of energy at position $\left(\mathrm{i}, \mathrm{j}\right.$ ) of each block (where the pixel value is $P_{i, j}$ ) is given by its gradient $\nabla P_{i, j}$ (equation 4). In textured image $\nabla P_{i, j}$ would be large at a large number of locations. A gradient-threshold $\lambda$ is taken to decide whether the gradient is larger than a certain limit and a count-threshold $C$ is taken to decide whether the block has an edge or is highly textured. All the blocks satisfying the constraint $I_{b} \in\{6,7,8\}$ are subjected to the test: a block is highly textured if it satisfies the equation (5).

$$
\begin{gathered}
\nabla P_{i, j}=a b s\left(p_{i+1, j}-p_{i, j}\right)+a b s\left(p_{i, j+1}-p_{i, j}\right) . \\
\|\left\{P_{i, j} \mid P_{i, j} \text { is a pixel and } \nabla P_{i, j}>\lambda\right\} \|>C . \\
J(i, j, b)=\hat{J}_{b}+\left\lfloor\frac{\left(\frac{G}{2}-P_{i, j, b}\right)^{2}}{\alpha}\right\rfloor .
\end{gathered}
$$

Where $\|\ldots\|$ is the cardinality of the set, $\alpha$ is predetermined constant, and $P_{i, j, b}$ is the pixel value at position $(i, j)$ of block $b$. Each block based on its corresponding $I_{b}$ is mapped to a JND value $\hat{J}_{b}, \hat{J}_{b} \in\{3,4, \ldots, 10\}$ while $I_{b} \in\{1,2, \ldots, 8\}$. We do one to one mapping for simplicity. We also consider pixel-luminance to have the final Just Noticeable Distortion (JND), $J(i, j, b)$, at any pixel position $(\mathrm{i}, \mathrm{j})$ in each textured block $\mathrm{b}$ (equation 6). It is actually incorporating the effect of luminance into JND value, since the distortion in an image is more noticeable in the mid-gray region and sensitivity changes parabolically as the gray value fluctuates on the both sides of midgray level. Note that $(i, j)$ means the element at row number $(i+1)$ and column number $(j+1)$ in a two dimensional array.

\section{The Proposed Lossless Watermarking Algorithm}

During the embedding process we find out the textured blocks in the original image $I$, then considering the HVS we extract the LSBs of the pixels in these textured blocks. The extracted LSBs are stored in a bit-string that is compressed losslessly to have 


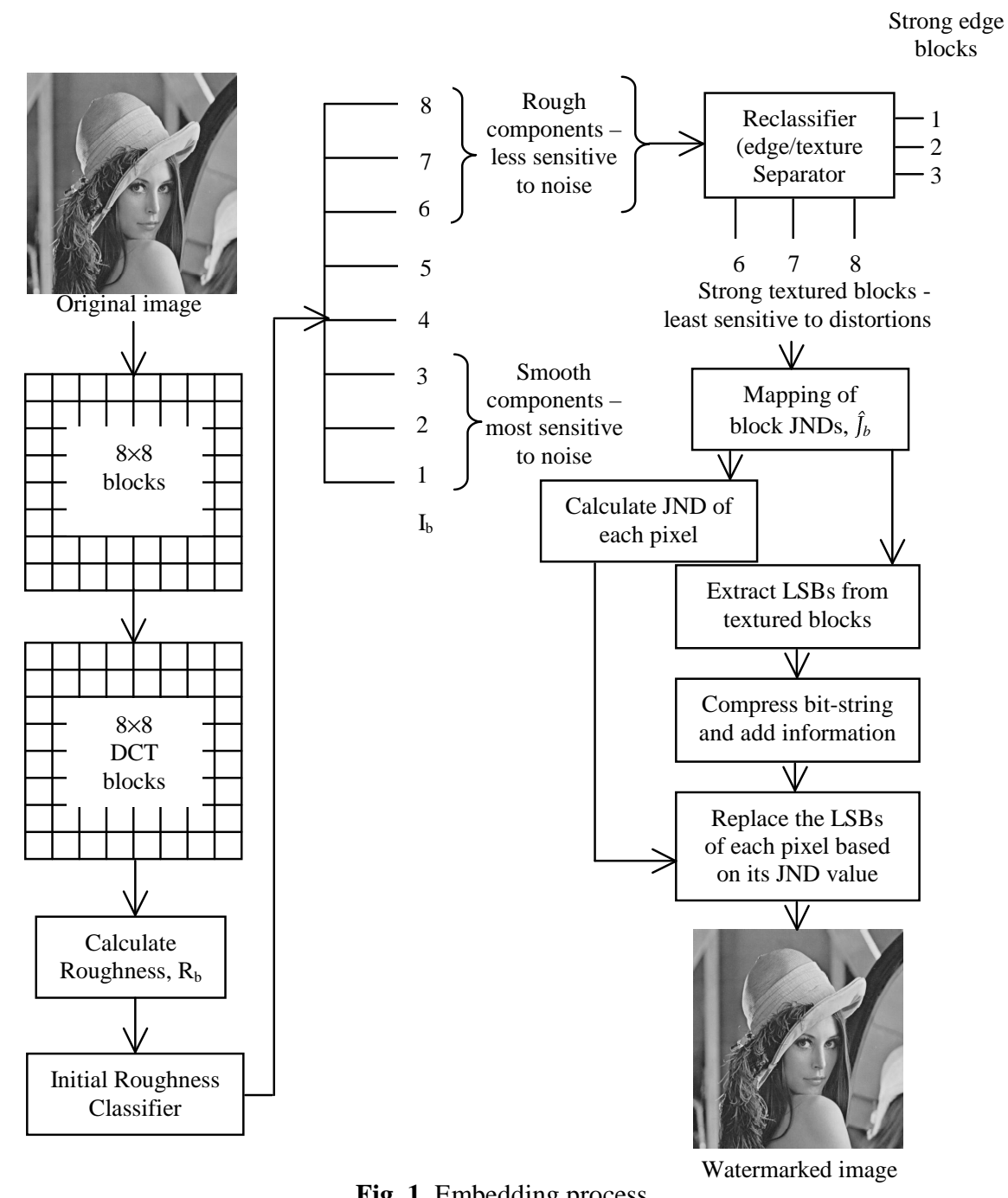

enough space to concatenate the authentication information, say the hash of the original image. We use arithmetic coding [8] for lossless compression. The resultant bit-string is called the watermark to be embedded into the original image. While embedding watermark we again consider the HVS to replace the LSBs of the pixels in the textured blocks. While extracting the LSBs of the pixels of textured blocks we try to minimize the amount of side information required. We find out the maximum JND value $\left(J_{\max , b}\right)$ for each block $\mathrm{b}$ and calculate the number of bits required to represent the $J_{\max , b}$ by bit $_{b}=\log _{2}\left(J_{\max , b}\right)$. So we extract bit $_{b}$ bits of LSBs per pixel of the textured block $\mathrm{b}$. For making the decoding process easy we need to add some side information (unchanged pixel-indexes) with the watermark. Also we replace some LSBs (original LSBs are added to the watermark before compression) of first few blocks (say 10) to pass the initial side information (textured block-numbers, number of LSBs extracted 
per pixel of each block, watermark-length) to initiate the decoding process. In words the embedding process (Fig.1) includes the following steps:

- Divide the original image into $8 \times 8$ blocks

- Calculate the DCT of each $8 \times 8$ block

- Calculate the roughness of each block

- By using initial classifier separate the rough components (blocks) that are less sensitive to noise

- Reclassify the rough components to separate the high textured blocks from the strong edge blocks

- Map the block JNDs, $\hat{J}_{b}$

- Calculate the JND of each pixel in textured blocks

- Calculate the number of LSBs, $b i t_{b}=\log _{2}\left(J_{\max , b}\right)$, to be extracted from each block b

- Extract the bit $_{b}$ LSBs per pixel of textured block $\mathrm{b}$ and store in a bit-string

- Compress the bit-string losslessly

- Calculate the hash of the original image and concatenate it (together with other information as required) with the compressed bit-string

- While replacing $b i t_{b}$ bits of LSBs per pixel of block b with $b_{i t}$ bits of watermark: let $x_{1}=$ decimal value of $b_{i t}$ LSBs of a pixel value at position $(\mathrm{i}, \mathrm{j}) ; x_{2}=$ decimal value of $b_{i t}$ bits of watermark to be embedded, $J(i, j, b)=$ JND value of pixel at position (i,j). If $\left(a b s\left(x_{2}-x_{1}\right) \leq J(i, j, b)\right)$ then replace the $b i t_{b}$ LSBs of this pixel with $b_{i t}$ bits of watermark, else keep the pixel unchanged.

Note that we do replacement of the LSBs of pixels (of textured blocks) instead of adding the watermark data. This leaves only a few or no pixels without embedding data. That is why the amount of side information of indexes of pixels without embedding is very low.

During decoding we extract the payload using the side information. After separating the authentication information we decompress the compressed bit-string of LSBs. Finally we replace the LSBs of the pixels of the textured blocks using the side information to get the exact copy of the original image. We calculate the hash of the reconstructed image and compare the calculated hash to the extracted hash.

\section{Experimental Results and Discussion}

We first present here our experimental results and then compare the results with other algorithms proposed so far.

\subsection{Experimental Results}

We have implemented our algorithm and applied to several images of different types: medical, astronomical, and general images used in the literature. We have used 
8 Mohammad Awrangjeb et al.

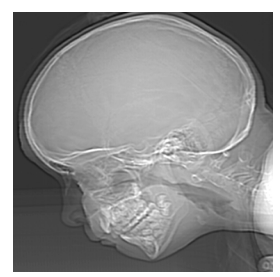

Fig. 2. CAT $(374 \times 452)$

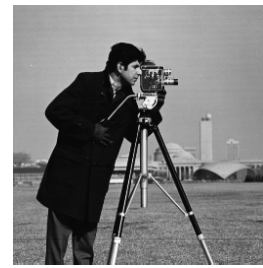

Fig. 5. Man $(256 \times 256)$

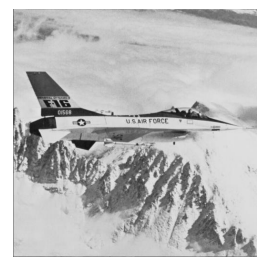

Fig. 8. Airplane $(512 \times 512)$

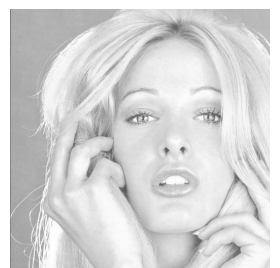

Fig. 11. Tiffany $(512 \times 512)$

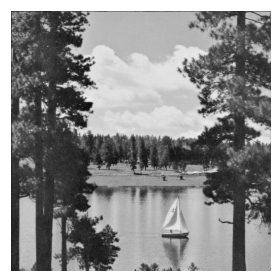

Fig. 14. Sailboat $(512 \times 512)$
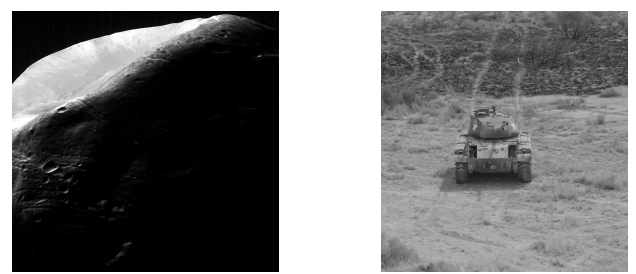

Fig. 3. Mars Moon $(683 \times 1000)$

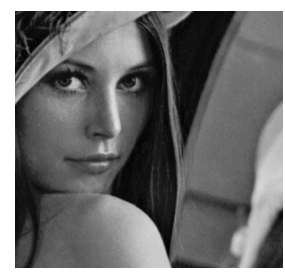

Fig. 6. Lena $(300 \times 300)$

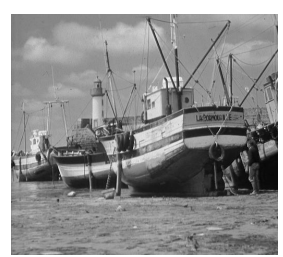

Fig. 9. Boat $(512 \times 512)$

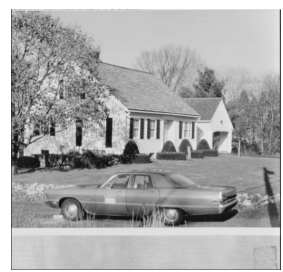

Fig. 12. House $(512 \times 512)$

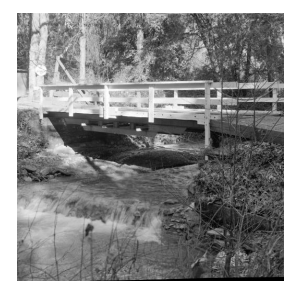

Fig. 15. Bridge $(512 \times 512)$
Fig. 4. Tank $(512 \times 512)$

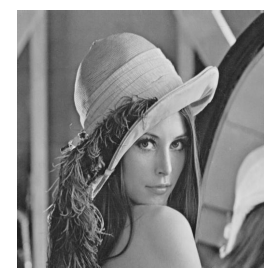

Fig. 7. Lena $(512 \times 512)$

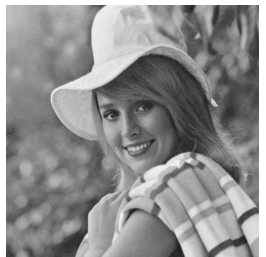

Fig. 10. Elaine $(512 \times 512)$

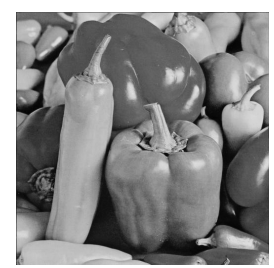

Fig. 13. Peppers $(512 \times 512)$

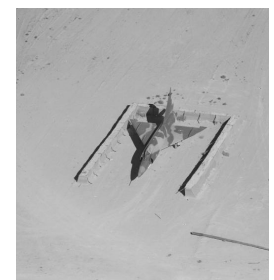

Fig. 16. Jet $(512 \times 512)$ 
arithmetic coding for compression [8]. Table 1 shows the experimental results we have found for our proposed algorithm. All images are 8-bit grayscale. These results show that the proposed algorithm offers reversible data hiding with higher capacity and imperceptible artifacts.

\subsection{Comparison with other Algorithms}

First we reveal limitations of the recent proposed lossless watermarking algorithms. Lossless compression and encryption of bit plane method by Fridrich et al. [3] has the limitations: (i) capacity is not very high, and (ii) higher payload forces the method to embed watermark at higher bit-planes and hence the artifacts are easily visible. Other watermarking methods proposed by Fridrich et al. in [4], [7], [9], [10] either offer limited capacity or create artifacts that are easily visible. Another problem is that these algorithms do not offer enough capacity after lossless compression for images of smaller sizes. Reversible data hiding method by Celik et al. [1] gives high capacity.

Table 1. Experimental results of the proposed algorithm

\begin{tabular}{|c|c|c|c|}
\hline no & Image $($ width $\times$ height $)$ & PSNR $(\mathrm{dB})$ & Capacity (byte) \\
\hline 1 & CAT $(374 \times 452)$ & 44.46 & 5521 \\
\hline 2 & Mars moon $(683 \times 1000)$ & 41.90 & 1078 \\
\hline 3 & Tank $(512 \times 512)$ & 37.93 & 379 \\
\hline 4 & Man $(256 \times 256)$ & 37.49 & 775 \\
\hline 5 & Lena $(300 \times 300)$ & 37.72 & 594 \\
\hline 6 & Lena $(512 \times 512)$ & 38.98 & 1901 \\
\hline 7 & Airplane $(512 \times 512)$ & 39.38 & 3656 \\
\hline 8 & Boat $(512 \times 512)$ & 40.69 & 1939 \\
\hline 9 & Elaine $(512 \times 512)$ & 42.79 & 2140 \\
\hline 10 & Tiffany $(512 \times 512)$ & 36.38 & 5087 \\
\hline 11 & House $(512 \times 512)$ & 37.72 & 3981 \\
\hline 12 & Pepper $(512 \times 512)$ & 38.70 & 3218 \\
\hline 13 & Sailboat $(512 \times 512)$ & 36.56 & 1261 \\
\hline 14 & Bridge $(512 \times 512)$ & 39.80 & 676 \\
\hline 15 & Jet $(512 \times 512)$ & 44.86 & 5238 \\
\hline-- & Average & 39.69 & 2496 \\
\hline
\end{tabular}

But the fundamental limitation with this method is: images that do not give enough data for losslessly compression, the embedding level becomes larger and the artifacts are too much visible. For example this method does not give enough capacity (at least 128 bits) for images $1,7,9,10,13$ in table 1 while we use compression algorithms like LZW [11], JBIG [21] or arithmetic coding [8] even if we increase the embedding level up to 50. Celik et al. suggest to use CALIC [16] lossless algorithm. But if we increase the embedding level up to 50 for image Tiffany we get only 256 bytes of data. It is impossible to compress such small amount of data to have enough space (at 
least 16 bytes) for embedding information. Moreover, amount of distortion is at most 50, so artifacts are easily visible. Methods proposed in [5], [12], [13] though offer high capacity, artifacts are visible. These algorithms also offer limited capacities for images of smaller sizes.

Recently, two lossless watermarking algorithms have been proposed [14], [15]. The method in [15] is especially for palette images. In [14] the capacity is limited by the frequency of the most frequent gray-level in the image. We provide some data to compare [14] with our proposed algorithm (Table 2).

Table 2. Comparing capacity and PSNR offered by [14] and the proposed algorithm

\begin{tabular}{|c|c|c|c|c|c|}
\hline \multirow{2}{*}{ no } & Image & \multicolumn{2}{|c|}{ Capacity (byte) } & \multicolumn{2}{c|}{ PSNR (dB) } \\
\cline { 3 - 6 } & $(512 \times 512)$ & {$[14]$} & Proposed & {$[14]$} & Proposed \\
\hline 1 & Airplane & 2022 & 3656 & 48.3 & 39.38 \\
\hline 2 & Lena & 683 & 1901 & 48.2 & 38.98 \\
\hline 3 & Tiffany & 1098 & 5087 & 48.2 & 36.38 \\
\hline 4 & Jet & 7498 & 5238 & 48.7 & 44.86 \\
\hline 5 & Pepper & 681 & 3218 & 48.2 & 38.7 \\
\hline 6 & Sailboat & 913 & 1261 & 48.2 & 36.56 \\
\hline 7 & House & 1789 & 3981 & 48.3 & 37.72 \\
\hline- & Average & 2098 & 3478 & 48.3 & 38.94 \\
\hline
\end{tabular}

The method in [14] offers a fixed PSNR 48.13 for all images because of its embedding criteria (shifting of histogram). We found that the claimed embedding of $60 \mathrm{k}$ bits of data by [14] was not always guaranteed. It was possible for the image Jet, which is a special case due to the flat nature of the image. If we exclude the Jet image, table 2 indicates that [14] offers an average of $9.5 \mathrm{k}$ bits of embedding capacity with a average (constant) PSNR 48.23dB, whereas our proposed method gives average embedding capacity $25.5 \mathrm{k}$ bits with average PSNR $37.95 \mathrm{~dB}$. Including the image Jet the proposed algorithm offers higher average capacity (28k bits) than [14] (17k bits). The average values including the Jet image are shown in Table 2, where the capacities are in bytes. We try to exclude the smooth images (like the image Jet) into account here because they always offer enough embedding capacity.

The lossless compression and encryption of bit-plane(s) by Fridrich et al. [3] offers low capacity for two reasons: (i) since bits at LSB positions are truly random, they do not offer a good compression ratio, and (ii) the amount of data to be compressed is very low; for a $512 \times 5128$-bit image we get only $4 \mathrm{kB}$ data if only 1 key bit-plane is selected. The RS-vector method [4] divides pixels into $R, S, U$ groups. $U$ groups are unusable, however it embeds only 1 bit per group. If each group contains 4 pixels and there is no $U$ group at all, a $512 \times 5128$-bit image can embed maximum $8 \mathrm{kB}$ data. On the other hand, our proposed method selects $8 \times 8$ blocks based on image nature and embeds 256 bits data per block on the average. To have the same capacity like RSvector method mentioned above we need only 256 blocks on the average out of 4096 blocks for a $512 \times 5128$-bit image. 
Hence, the experimental results show that our proposed algorithm is better, since it gives higher capacity for almost all images and better PSNR compared to other methods proposed so far. The most important aspect of our proposed algorithm is the consideration of the HVS during embedding, and hence the artifacts are completely imperceptible. Thus the proposed algorithm fulfills the requirements (invisible distortions and higher capacity) of lossless watermarking technique and is better than other existing algorithms in the literature.

\section{Conclusions}

We present here a lossless watermarking technique with a higher embedding capacity. By considering the Human Visual System (HVS) we leave no pixel into the watermarked image with a distortion greater than the limit determined by the JND value of that pixel. Hence, there is no visible distortion at all in the watermarked image by our proposed algorithm. The experimental results prove that the proposed algorithm is better than any other algorithm proposed so far in the lossless watermarking literature. Additional security against active attacks can be obtained by encrypting the watermark bit-string with a secret key $K$ before embedding. The proposed method can also be used for color images. It works on both smooth and textured images. It also works on small images as well as large images. The proposed method is simpler, gives higher average capacity with no perceptual distortion. Hence, we believe that the proposed method will be useful in lossless watermarking applications concerning medical, astronomical, and military imagery.

\section{References}

1. Celik, M.U., Sharma, G., Tekalp, M.A., Saber, E.: Reversible Data Hiding. In Proceedings of International Conference on Image Processing, vol. 2. Rochester, NY, USA (24 Sep 2002) $157-160$

2. Honsinger, C.W., Jones, P.W., Rabbani, M., Stoffel, J.C.: Lossless Recovery of an Original Image Containing Embedded Data. US Patent \# 6,278,791 (Aug 2001)

3. Fridrich, J., Goljan, M., Du, R.: Invertible Authentication. In Proc. of SPIE Photonics West, Security and Watermarking of Multimedia Contents III, vol. 3971. San Jose, California, USA (Jan 2001) 197-208

4. Fridrich, J., Goljan, M., Du, R.: Lossless Data Embedding - New Paradigm in Digital Watermarking. Special Issue on Emerging Applications of Multimedia Data Hiding, vol. 2. (Feb 2002) 185-196

5. Tian, J.: Wavelet-based reversible watermarking for authentication. In Proc. Security and Watermarking of Multimedia Contents IV, Electronic Imaging 2002, vol. 4675. (20-25 Jan 2002) 679-690

6. Kankanhalli, M.S., Rajmohan, Ramakrishnan, K.R.: Content-based Watermarking of Images. In Proceedings of 6th ACM International Multimedia Conference. Bristol, UK (Sep1998) 61-70

7. Fridrich, J., Goljan, M., Du, R.: Lossless Data Embedding for all Image Formats. In Proc. SPIE Photonics West, Electronic Imaging, Security and Watermarking of Multimedia Contents, vol. 4675. San Jose, California, USA, (Jan 2002) 572-583 
8. Sayood, K.: Introduction to Data Compression. 2nd edn. Morgan Kaufmann (2000) 77-104

9. Goljan, M., Fridrich, J., Du, R.: Distortion-free Data Embedding. In 4th Information Hiding Workshop, LNCS, vol. 2137. Springer-Verlag, New York, USA (2001) 27-41

10. Fridrich, J., Goljan, M., Du, R.: Invertible Authentication Watermark for JPEG Images. In Proc. of Information Technology: Coding and Computing. Las Vegas, Nevada, USA, (2-4 Apr 2001) 223-227

11. Gonzalez, R.C., Woods, R.E.: Digital Image Processing. 2nd edn. Pearson Education International, Prentice Hall (2002) 446-448

12. Vleeschouwer, C.D., Delaigle, J.F., Macq, B.: Circular Interpretation of Histogram for Reversible Watermarking. In IEEE 4th Workshop Multimedia Signal Processing. (2001) $345-350$

13. Xuan, G., Chen, J., Zhu, J., Shi, Y.Q., Ni, Z., Su, W.: Lossless Data hiding based on Integer Wavelet Transform. In IEEE International Workshop on Multimedia Signal Processing. Marriott Beach Resort St. Thomas, US Virgin Islands (9-11 Dec 2002)

14. Ni, Z., Shi, Y.Q., Ansari, N., Su, W.: Reversible Data Hiding. In: Proceedings of International Symposium on Circuits and Systems (ISCAS 2003), vol. 2. Bangkok, Thailand (25-28 May 2003) 912-915

15. Hongmei, L., Zhefeng, Z., Jiwu, H., Xialing, H., Shi, Y.Q.: A High Capacity Distortion-free Data Hiding Algorithm for Palette Image. In: Proceedings of International Symposium on Circuits and Systems (ISCAS 2003), vol. 2. Bangkok, Thailand (25-28 May 2003) 916-919

16. WU, X.: Lossless Compression of Continuous-Tone Images via Context Selection, quantization, and modeling. In: IEEE Transactions on Image Processing, vol. 6, no. 5. (May 1997) $656-664$

17. Tian, J.: Reversible Watermarking by Difference Expansion. In Proceedings of Workshop on Multimedia and Security, ACM, Juan-les-Pins, France (Dec 2002) 19-22

18. MACQ, B.: Lossless Multiresolution Transform for Image Authenticating Watermarking. In Proceedings of EUSIPCO, Tempere, Finland, (Sep 2000)

19. Vleeschouwer, C.D., Delaigle, J.F., Macq, B.: Circular Interpretation of Bijective Transformations in Lossless Watermarking for Media Asset Management. In IEEE Transaction on Multimedia, (Mar 2003)

20. Bender, W., Gruhl, D., Morimoto, N., LU, A.: Techniques for Data Hiding. In IBM Systems Journal, vol. 35, no. 3-4. (1996) 313-336

21. Sayood, K.: Introduction to Data Compression. 2nd edn. Morgan Kaufmann (2000) 106114 